$$
\text { "hoffmann" — 2008/4/9 — 10:06 — page 385 — \#1 }
$$

\title{
Typical mistakes in Mental Cutting Test and their consequences in gender differences
}

\author{
Brigitta Németh, Csilla Sörös and Miklós Hoffmann
}

Abstract. Spatial ability of first year university students is measured and evaluated in this paper. We used standard Mental Cutting Test (MCT), where a body is given by perspective view and correct cross section has to be chosen. While gender differences in MCT are reported by several papers including our earlier results, much less known are the reasons of these differences. Here we show that typical mistakes (answers to problems which are close to be correct) can be one of the possible reasons, since female students made typical mistakes in some cases more frequently than males.

Key words and phrases: spatial ability, Mental Cutting Test, gender differences, typical mistakes.

ZDM Subject Classification: G80, G10, D70, C30, 51N05.

\section{Introduction}

Spatial ability and its measurement is of central importance in technical higher education. According to McGee, the most accepted definition of spatial ability is "the ability to mentally manipulate, rotate, twist or invert pictorally presented stimuli" [1]. The five components of spatial skills are spatial perception, spatial visualization, mental rotations, mental relations, spatial orientation.

While the usual way to improve this ability is to give descriptive geometry courses in every technical faculty in Hungary, the importance of the evaluation of this ability is somehow underestimated, however standardized tests have been

Copyright (C) 2007 by University of Debrecen 


$$
\text { "hoffmann" — 2008/4/9 — 10:06 — page 386 — \#2 }
$$

developed decades ago, among which Mental Rotations Test (MRT) and Mental Cutting Test (MCT) are of greatest importance. Mental Rotation Test is introduced by Vandenberg and Kuse [2], while Mental Cutting Test, originally developed for entrance examination in the United States [3], has a long history and widely used for testing the spatial ability of students at any level. Objective Test on Orthographic Projection (OTO) evaluates the effects of the education in orthographic view [4]. Space Imagination Test (TPP) has been developed by Juščaková and was standardized by an international cooperation in VEGA project $[5]$.

The aim of this paper is to evaluate classical MCT test results of first-year engineering students in Faculty of Engineering, Szent István University, Hungary, with special emphasis on gender differences. In [6] the authors published overall results of these tests where gender differences were found to be significant. Here we attempt to find possible reasons of this difference, concluding, that typical mistakes play central role in it.

In Section 2 we give a short overview of previous results, while in Section 3 typical mistakes are studied in details. Conclusion and further research directions close the paper in Section 4.

\section{Mental Cutting Test and gender differences}

In our project we used the standard Mental Cutting Test, which consists of 25 problems. In each problem perspective drawing of a solid body is given, which has been cut by a plane (c.f. Fig. 1). Students are asked for choosing the cross section among the 5 given alternatives, always one being correct.

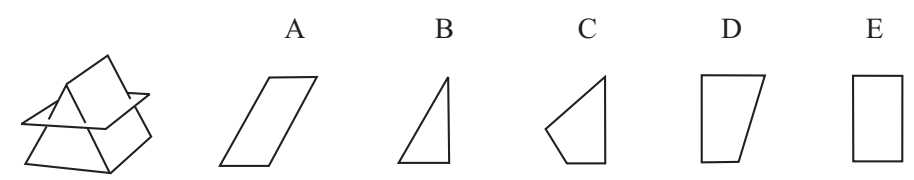

Figure 1. An example of MCT problems (the correct answer is E).

As it is already reported in several papers, female students generally achieve significantly less points in MCT: see national and international projects in [5], [7], [8], [9], [10], and also a longitudinal research by Gorska [11]. Beside classical measurement, MCT test in a stereographic circumstances was also used for female students by Tsutsumi et al. [7] with similar results. As Tsutsumi declares 


$$
\text { "hoffmann" — 2008/4/9 — 10:06 — page 387 — \#3 }
$$

laconically in [7], females "are much less likely to get high scores in the standard MCT".

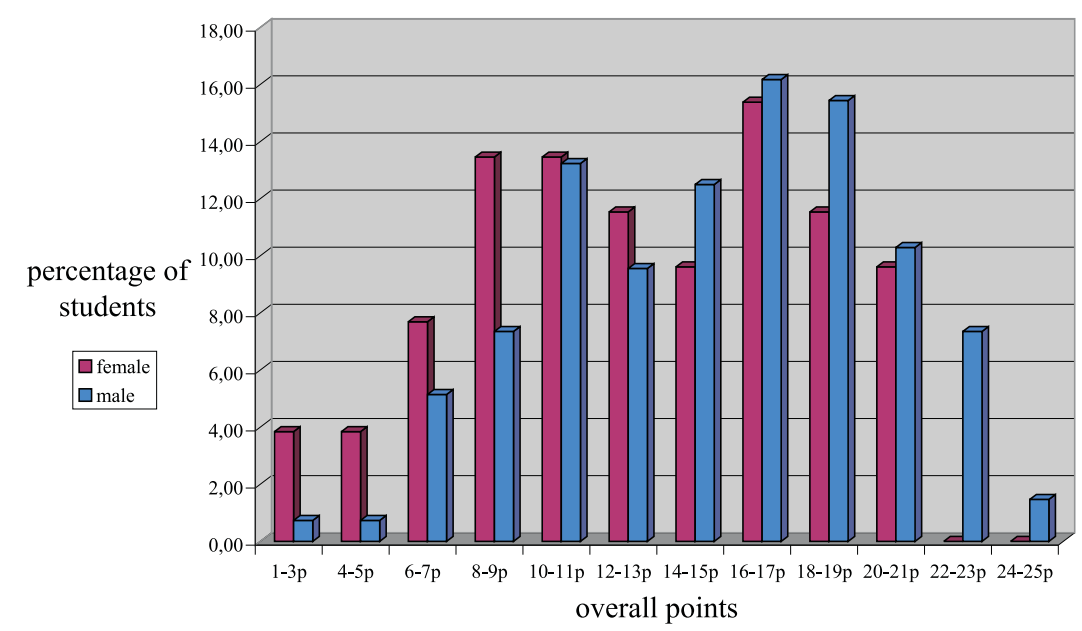

Figure 2. Detailed results of male and female students.

In our project the classical MCT test is used for first-year engineering students of Szent István University. The test has been filled by more than 250 students, approximately third of them being female students. Detailed results can be seen in Fig. 2. As one can easily observe from this diagram, scores of female students are strictly lower than that of male students. Especially remarkable the difference in the highest portion: among the students with best spatial skills the number of females are extremely low. Actually the female student with best result had 21 points, just falling into the highest part. The same difference can be seen in the lowest interval (1-5 points), but in the opposite meaning: less than $2 \%$ of male students achieved 5 or less points, while amongst female students this rate was more than $7.5 \%$. Further examination and statistical analysis of these results can be found in $[6]$.

\section{Typical mistakes - a possible reason of gender differences}

As we have seen in the previous section, several projects and researchers reported the gender differences in MCT results, including our Hungarian project 


$$
\text { "hoffmann" — 2008/4/9 — 10:06 — page 388 — \#4 }
$$

as well. The sources of this difference, however are not quite obvious. Here we attempt to find a possible reason for it.

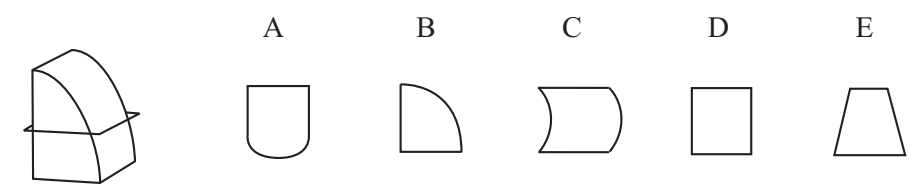

Figure 3. Curved objects usually yield curved cuts as typical mistake (the correct answer is $\mathrm{D}$, the typical mistake is $\mathrm{A}$ )

For each problem in the test, beside the only correct solution students can find four possible wrong alternatives as well. Evaluating the statistical results of the tests we observed that the wrong alternatives are not evenly distributed among those students who gave wrong answer to a certain problem. In almost all of the problems there is one alternative (wrong) cross section beside the correct solution, which has been chosen by relatively high percentage of students. This alternative (wrong) answer is called typical mistake.

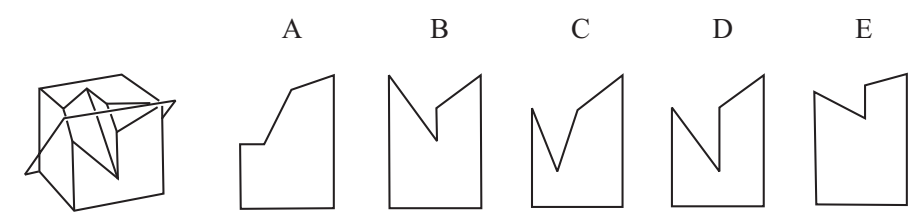

Figure 4. A difficult problem: similar cuts with slight metric differencies (the correct answer is E, the typical mistake is D)

Typical mistakes are obviously close to the correct answers in terms of their shape and metrical relations (angles and relative lengths). In some problems typical mistake can also arise from the unusual shape of the object: if the body is curved then we somehow expect curved cuts, thus several students choose the wrong curved intersection (c.f. Fig. 3). Tsutsumi et al. also supposed in [7], that failures are mostly based on the fact that students cannot recognize correctly the spatial form of the object.

Problems in MCT have been classified in [12] as pattern recognition problems and quantity problems. In the first category, like the problem in Fig. 3, one can find strongly different alternatives of possible cross sections, thus the right solution can be found simply by recognizing the pattern of the section from the spatial figure. In the quantity problems, however, some of the cross sections are similarly 


$$
\text { "hoffmann" — 2008/4/9 — 10:06 — page 389 — \#5 }
$$

structured to the correct one, thus the right answer can be determined only by guessing the relative quantities, like ratios of lengths or angles between the edges (see e.g. Fig. 4). Obviously these latter problems are among the most difficult ones in MCT.

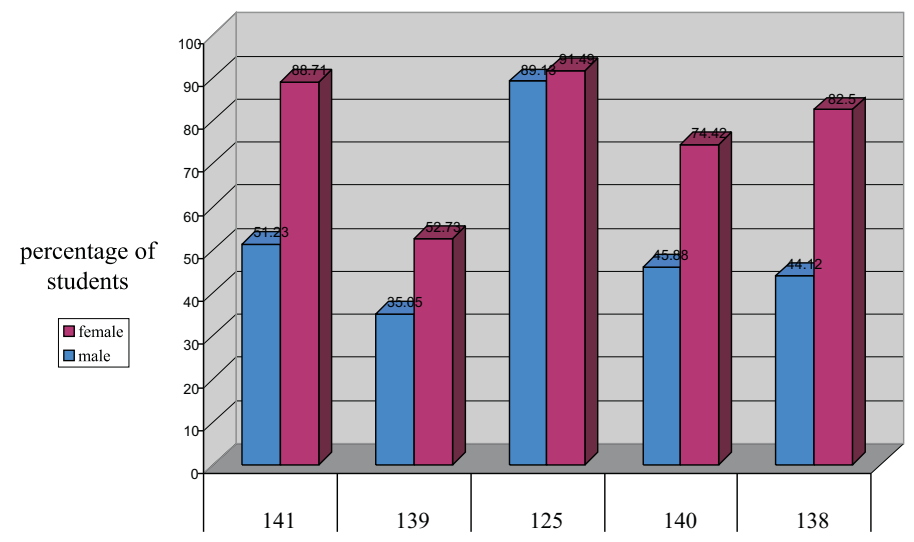

The five hardest problems

Figure 5. Choosing the typical mistake of the 5 hardest problems in percentage of students who failed the current problem (number of problems along the $x$-axis). Females chose typical mistakes more frequently than males.

Evaluating the test results we observed that in some cases female students choose typical mistakes more frequently than males. This difference was significant in the most difficult problems (the difficulty of the problem is measured by the percentage of correct answers), but surprisingly the difference was also obvious in the easiest problems. In problems of medium level we cannot observe significant difference between male and female students choosing typical mistakes. However the difference in the two sets of extremal (very easy and very hard) problems may partly explains the gender differences in MCT test.

In diagram of Fig. 5 we show the frequency of typical mistakes in the hardest problems. We have to emphasize that a very high percentage of female students failed to answer these problems. As we can see in the diagram, most of them chose the typical mistake. Possible reason of the failure is that in these hard problems the correct answer and the typical mistake can be distinguished by carefully observing very small metric differences. Most of the female students correctly judged the overall shape of the cross section, but failed to observe these 


$$
\text { "hoffmann" — 2008/4/9 — 10:06 — page 390 — \#6 }
$$

minor differences, while in terms of male students, if they found the overall shape, then they almost all found the correct metrical relations as well. If a male student failed these problems, then usually he had "no idea" at all even about the overall pattern of the cross section.

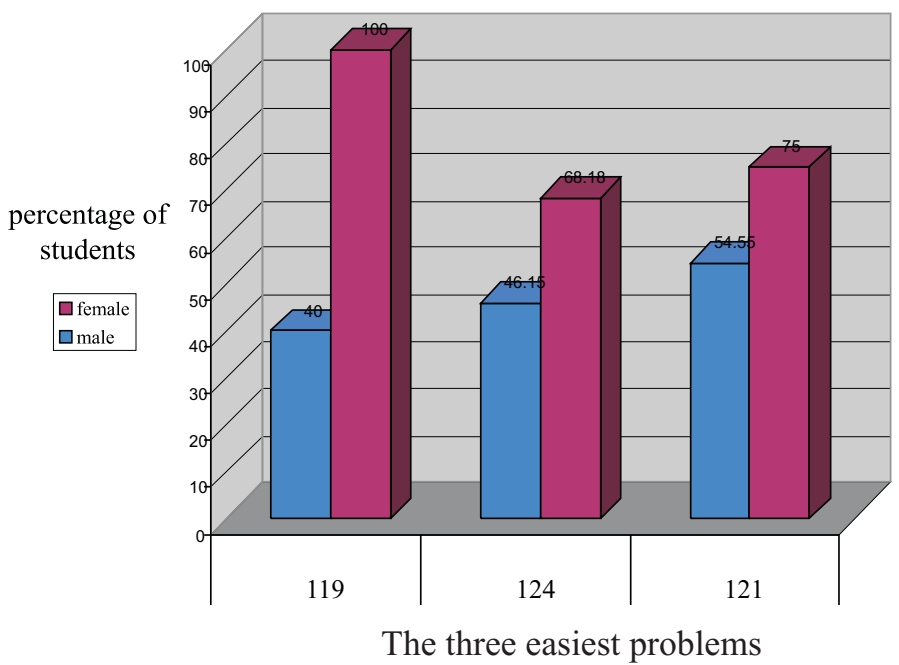

Figure 6. Choosing the typical mistake of the 3 easiest problems in percentage of students who failed the current problem (number of problems along the $x$-axis). Females chose typical mistakes more frequently than males here as well.

In diagram of Fig. 6 we show the frequency of typical mistakes in the easiest problems. This diagram also shows the dominancy of female students in chosing typical mistake. Note especially problem No. 119 where all the female students, who chose wrong answer, chose the typical mistake. These results may be explained simply by the fact that easy problems may not be studied carefully enough, however generally female students are more competent in this manner.

\section{Conclusion and further research}

Typical mistakes of Mental Cutting Test have been studied in this paper. Several research paper reported gender differences in the results of this test, but apart from general comments this fact remained unexplained in most of the cases. As we have observed in our research, female students choose typical mistake in 


$$
\text { "hoffmann" — 2008/4/9 — 10:06 — page 391 — \#7 }
$$

some problems more frequently, than male students, which can partly explain the gender differences in spatial ability test results. Further study of this topic in terms of other tests and longitudinal research of development of spatial ability are among our future aims in research.

\section{References}

[1] M. G. McGee, Human Spatial Abilities: Psychometric studies and environmental, genetic, hormonal and neurological influences, Psychological Bulletin 86, 899-918.

[2] S. G. Vandenberg, A. R. Kuse, Mental Rotations, a group test of three dimensional spatial visualization, Perceptual and Motor Skills 47 (1978), 599-604.

[3] CEEB Special aptitude test in spatial relations, College Entrance Examination Board, USA, 1939.

[4] K. Takeyama, R. Maeguchi, K. Chibana, K. Yoshida, Evaluation of Objective Test using a pair of orthographic projections for descriptive geometry, Journal for Geometry and Graphics 3 (1999), 99-109.

[5] Z. Juščaková, R. Gorska, A pilot study of a new testing method for spatial abilities evaluation, Journal for Geometry and Graphics 7 (2003), 237-247.

[6] B. Németh, M. Hoffmann, Gender differences in spatial visualization among engineering students, Annales Mathematicae et Informaticae 33 (2006), 169-174.

[7] E. Tsutsumi, K. Shiina, A. Suzaki, K. Yamanouchi, S. Takaaki, K. Suzuki, A Mental Cutting Test on female students using a stereographic system, Journal for Geometry and Graphics 3 (1999), 111-119.

[8] T. Saito, K. Shiina, K. Suzuki, T. Jingu, Spatial Ability Evaluated by a Mental Cut-

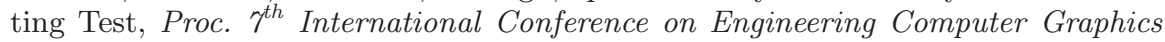
and Descriptive Geometry, Cracow, Poland (1996), 569-573.

[9] R. Gorska, S. Sorby, C. Leopold, Gender differences in visualization skills - an international perspective, The Engineering Design Graphics Journal 62 (1998), $9-18$.

[10] R. Gorska, S. Sorby, C. Leopold, International comparisons of gender differences in spatial visualization and the effect of graphics instruction on the development of these skills, Proc. of the $8^{\text {th }}$ Intl. Conf. of Engineering Comp. Graph. and Descriptive Geom. (ICECGDG), Austin, USA (1998), 261-266.

[11] R. Gorska, Spatial imagination - an overview of the longitudinal research at Cracow University of Technology, Journal for Geometry and Graphics 9 (2005), 201-208.

[12] E. Tsutsumi, A Mental Cutting Test using drawings of intersections, Journal for Geometry and Graphics 8 (2004), 117-126. 


$$
\text { "hoffmann" — 2008/4/9 — 10:06 — page 392 — \#8 }
$$

BRIGITTA NÉMETH and CSILLA SÖRÖS

DEPARTMENT OF DESCRIPTIVE GEOMETRY AND COMPUTER SCIENCE

SZENT ISTVÁN UNIVERSITY

BUDAPEST

HUNGARY

E-mail: Nemeth.Brigitta@ymmfk.szie.hu

E-mail: keleties@gmail.com

MIKLÓS HOFFMANN

INSTITUTE OF MATHEMATICS AND COMPUTER SCIENCE

KÁROLY ESZTERHÁZY COLLEGE

LEÁNYKA STR 4

H-3300, EGER

HUNGARY

E-mail: hofi@ektf.hu

(Received July, 2007) 